ARTICLE

\title{
Identification of relevant genetic alterations in cancer using topological data analysis
}

\author{
Raúl Rabadán (10 ${ }^{1 凶}$, Yamina Mohamedi ${ }^{2,3}$, Udi Rubin ${ }^{1,7}$, Tim Chu ${ }^{1}$, Adam N. Alghalith (1) ${ }^{4}$, Oliver Elliott ${ }^{1}$, \\ Luis Arnés ${ }^{1}$ Santiago Cal ${ }^{2,3}$, Álvaro J. Obaya ${ }^{3,5}$, Arnold J. Levine ${ }^{6 凶} \&$ Pablo G. Cámara (10 ${ }^{4 凶}$
}

Large-scale cancer genomic studies enable the systematic identification of mutations that lead to the genesis and progression of tumors, uncovering the underlying molecular mechanisms and potential therapies. While some such mutations are recurrently found in many tumors, many others exist solely within a few samples, precluding detection by conventional recurrence-based statistical approaches. Integrated analysis of somatic mutations and RNA expression data across 12 tumor types reveals that mutations of cancer genes are usually accompanied by substantial changes in expression. We use topological data analysis to leverage this observation and uncover 38 elusive candidate cancer-associated genes, including inactivating mutations of the metalloproteinase ADAMTS12 in lung adenocarcinoma. We show that $A D A M T S 12^{-/-}$mice have a five-fold increase in the susceptibility to develop lung tumors, confirming the role of ADAMTS12 as a tumor suppressor gene. Our results demonstrate that data integration through topological techniques can increase our ability to identify previously unreported cancer-related alterations.

\footnotetext{
${ }^{1}$ Departments of Systems Biology and Biomedical Informatics, Columbia University, 1130 St. Nicholas Ave., New York, NY 10032, USA. ${ }^{2}$ Departamento de Bioquimica y Biologia Molecular, Universidad de Oviedo, Oviedo, Asturias, Spain. ${ }^{3}$ IUOPA, Instituto Universitario de Oncologia, Oviedo, Asturias, Spain. ${ }^{4}$ Department of Genetics and Institute for Biomedical Informatics, Perelman School of Medicine, University of Pennsylvania, 3400 Civic Center Blvd., Philadelphia, PA 19104, USA. ${ }^{5}$ Departamento de Biologia Funcional, Universidad de Oviedo, Oviedo, Asturias, Spain. ${ }^{6}$ The Simons Center for Systems Biology, Institute for Advanced Study, Princeton, NJ 08540, USA. ${ }^{7}$ Present address: Memorial Sloan Kettering Cancer Center, 1275 York Ave, New York, NY 10065, USA.凶email: rr2579@cumc.columbia.edu; alevine@ias.edu; pcamara@pennmedicine.upenn.edu
} 
critical foundation for targeted cancer therapies is the identification of molecular mechanisms that are necessary for tumor development and maintenance. Large-scale cross-sectional cancer molecular studies, such as The Cancer Genome Atlas (TCGA) and the International Cancer Genome Consortium, enable this identification by systematically compiling genetic alterations across many tumors ${ }^{1,2}$. Tumors that present recurrently altered genes or pathways are suspected to be driven by common molecular mechanisms. By leveraging computational approaches that seek signatures of positive selection ${ }^{3}$, these studies have produced extensive catalogues of frequentlymutated, cancer-associated genes ${ }^{4}$. These studies have also revealed that most cancer mutations occur at low frequencies $(<10 \%$ of samples), including potentially actionable therapeutic targets ${ }^{4}$.

The identification of low-prevalence cancer-associated mutations using recurrence-based methods is challenging because of the large number of samples needed to achieve statistical power and the inherent complexity in modeling the background mutation rates. The rate of neutral mutations within a cancer type can dramatically differ among patients, genomic regions, or mutation types $^{3}$, limiting the power of recurrence-based methods. It is estimated that for the current size of ongoing cross-sectional studies (typically consisting of less than 1000 patients) only cancer-associated mutations that occur at intermediate or high frequencies $(>15 \%)$ are fully accessible to recurrence-based methods ${ }^{4}$. This is consistent with the observation that patients in these studies often completely lack mutations in known cancerassociated genes ${ }^{5}$. These results highlight the need of recurrencebased methods that can model rare events ${ }^{6,7}$ or, alternatively, methods for the identification of cancer-associated genes that are not based on recurrence.

An approach to the identification of cancer-associated genes that is not based on modeling the mutation rate is the integration of other types of data from the tumor ${ }^{8,9}$. If a mutation is accompanied by consistent changes in copy number, gene expression, and/or methylation, it is possible to leverage these changes to relate the mutation event to cancer progression. Several studies have utilized changes in the copy number, expression, or methylation of the mutated gene (cis-effects) to identify novel cancer-associated mutations ${ }^{10-12}$. However, the identification of cancer-associated mutations based on changes in genes other than the mutated gene (trans-effects) is more challenging and usually requires providing information about known gene-gene relationships to reduce the number of false positives. DriverNet ${ }^{13}$, OncoIMPACT ${ }^{14}, \mathrm{CaMoDi}^{15}$, and $\mathrm{Xseq}^{16}$ utilize trans-effects in gene expression to identify cancer-associated mutations. To limit the dimensionality of the expression space, these methods use expression modules (sets of co-expressed genes, functionally related genes, or gene networks). However, an approach that fully takes into account the complexity of the expression space is currently lacking.

Current approaches for the identification of cancer-associated mutations using expression data are sensitive to several confounding effects. Genomic regions with open chromatin can be more easily accessed by DNA repair enzymes, leading to anticorrelations between gene expression levels and mutation rates ${ }^{3}$. Furthermore, tumors with different expression signatures, such as genomically unstable tumors, can have different mutation rates ${ }^{17,18}$. These effects lead to spurious correlations between mutations and expression signatures and are a source of false positives for current algorithms.

To address these problems, we devise an approach to identify cancer-associated mutated genes using expression data from multiple tumors. Our approach makes use of topological data analysis $^{19,20}$ (TDA) to reconstruct the structure of the expression space, and takes into account the above spurious effects when assessing the significance of a mutated gene. Its application to mutation and expression data of 4476 patients from 12 tumor types leads to the identification of 95 mutated cancer genes, out of which 38 are previously unreported low-prevalence genes (average prevalence within the same tumor cohort $=5 \%$ ). We hence propose a complementary approach to recurrence-based methods, enabling the identification of elusive, but potentially clinically-relevant, mutated cancer genes.

\section{Results}

Topological representation of low grade glioma expression. The expression profile of a tumor can be mathematically described as a point in a high-dimensional expression space, where each dimension represents the mRNA level of a gene and the dimensionality of the space is given by the number of expressed genes. Points that lie close to each other in this space correspond to tumors with similar expression profiles. The set of all possible tumors of a cancer type spans a subspace of the expression space. Measuring the expression profiles of individual tumors in a cross-sectional study is equivalent to sampling a finite set of points from this subspace.

We considered 513 primary low grade glioma (LGG) tumors from TCGA for which both RNA-seq and whole-exome DNAseq data were available ${ }^{21}$. To infer the structure of the expression space of LGG from this RNA-seq data, we used a topological approach ${ }^{19,20}$. Topology is the mathematical field that studies how different parts of a space are connected to each other. TDA generalizes some of the notions of topology to sets of points and pairwise distances. Thus, TDA aims to infer and summarize the topological structure of a space given only a finite sample of points. TDA has been recently used to study viral reassortment ${ }^{22}$, human recombination ${ }^{23,24}$, cell differentiation ${ }^{25}$, breast cancer ${ }^{26}$, and other complex genetic diseases ${ }^{27}$.

We used the TDA algorithm Mapper ${ }^{28}$ to build a lowdimensional representation of the expression space of LGG using the expression data of the TCGA cohort (Fig. 1a). Mapper generates a network representation of the expression space, in which each node corresponds to a set of tumors with similar expression profile. A given tumor can appear in more than one node, and if two nodes have one or more tumors in common they are connected by an edge. Contrary to other methods for dimensionality reduction, such as principal component analysis and multidimensional scaling ${ }^{29}$, the topological representations produced by Mapper preserve local relationships of the highdimensional expression space. Any two tumors close to each other in the topological representation (as measured by the number of edges contained in the shortest path that connects the two tumors) are ensured to be close to each other in the original high-dimensional expression space. We used Pearson's correlation as a measure of similarity between the expression profiles of individual tumors. The topological representation of the LGG expression space consisted of three regions (Fig. 1a), consistent with the expression subtypes found in clustering analyses ${ }^{21}$. These regions, however, were bridged by thin structures in the topological representation, indicating that some tumors have an expression profile characteristic of multiple expression subtypes (Fig. 1a).

Identification of cancer-associated genes in LGG. We hypothesized that if a mutated gene appears localized in the expression space, it is associated with consistent global expression patterns across a subset of tumors, and is therefore a candidate driver of tumor progression (Fig. 1b). On the other hand, if mutations of a gene are clonally expanded as a result of being in the same 

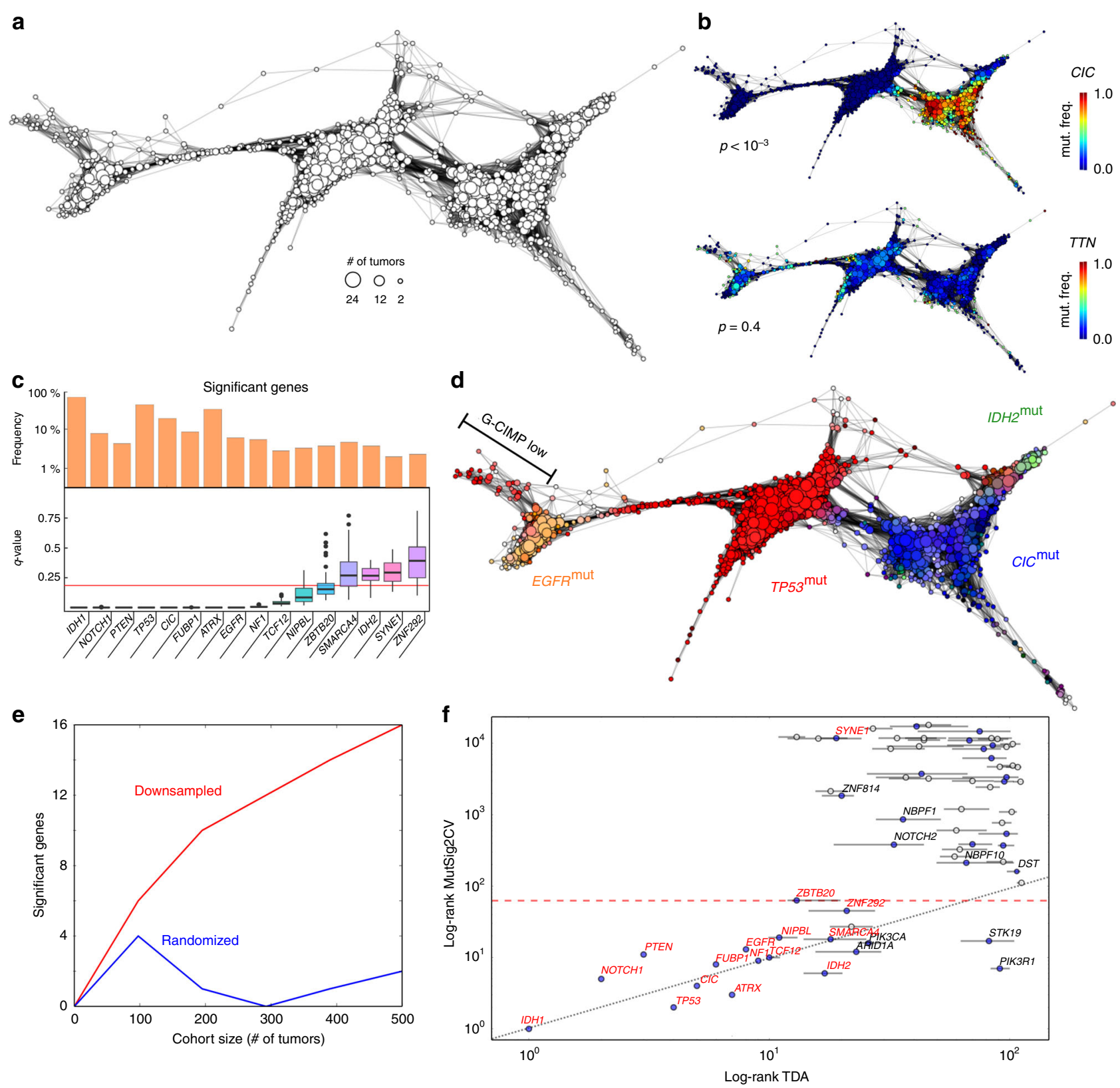

genome as a positively-selected mutation, but are not cancerrelated, they will appear randomly scattered in the expression subspace (Fig. 1b).

To test this hypothesis, we implemented a computational approach that assesses the localization of nonsynonymous somatic mutations in the expression space of tumors (Methods). To control for the presence of spurious correlations between the mutation rate and the tumor expression profile, we assessed the localization of the mutational tumor burden (defined as the total number of somatic mutations in each tumor) in the reconstructed expression space (Supplementary Fig. 1a, Methods). Based on this analysis, we subsampled mutations in two hypermutated tumors $\left(\mathrm{n}_{\text {mut }}>10^{2.5}\right)$ that were present in the LGG cohort. In addition, we assessed the similarity between the expression and the mutation profile of each individual gene in the reconstructed expression space (Methods). After correcting for these spurious correlations, 16 mutated genes were significantly localized in the reconstructed expression space of LGG (Fig. 1c, $q$-value $<0.15$, Benjamini-Hochberg procedure). These included well-known high-prevalence $(>15 \%)$ driver genes, like IDH1, TP53, ATRX, and $C I C$, in addition to several low-prevalence mutated genes, like NIPBL (mutated in $4 \%$ of the tumors) and ZNF292 (mutated in $3 \%$ of the tumors), which have been recently reported in a larger cohort of gliomas ${ }^{21}$. In total, 15 out of the 16 significant mutated genes were previously reported ${ }^{21,30}$, with SYNE1 (mutated in $2 \%$ of the tumors) the only new candidate. We did not observe a significant correlation between the significance and prevalence of statistically significant genes (Pearson's correlation coefficient between prevalence and $q$-value, $r=-0.34$, $p$-value $=$ 0.19). In particular, some of the most significant genes according to our approach, like FUBP1, NOTCH1, PTEN, EGFR, and NF1, were mutated in less than $10 \%$ of the patients within that tumor type (Fig. 1c), indicating that mutations in these genes are strongly associated with global changes in expression. These results were stable across the parameter space of the Mapper algorithm (Fig. 1c, Supplementary Fig. 1b, Methods).

The location of significant genes in the reconstructed expression space of LGG was consistent with the known molecular subtypes of adult diffuse gliomas ${ }^{21}$ (Fig. 1d, Supplementary Fig. 2). Of particular note, IDH2-mutant tumors were 
Fig. 1 Identification of mutated cancer genes in LGG using an integrative topological approach. a Topological representation of the expression space of LGG based on the expression data of 513 tumors. Each node represents a set of tumors with similar global expression patterns. The size of each node represents the number of tumors in the set. Edges connect nodes that share at least one tumor. Three large expression groups are clearly visible in the representation. $\mathbf{b}$ The localization of each mutated gene in the topological representation is assessed statistically. Mutated genes significantly localized in the expression space are candidate drivers of tumor progression. The topological representation of the expression space of LGG labeled by the frequency of somatic mutations of the CIC (top) and TTN (bottom) genes is displayed as an example. CIC mutations are significantly localized in the expression space of LGG (permutation test $p$-value $<10^{-3}, n=10^{3}$ permutations), consistently with being a driver of tumor progression. $\mathbf{c}$ List of significantly (permutation test, Benjamini-Hochberg $q$-value $<0.15 n=10^{4}$ permutations) localized mutated genes in the reconstructed expression space of LGG. The prevalence of mutations in the cohort and the distribution of the statistical significance across the parameter space of the topological representation are also displayed. Box-plot elements: center line, median; box limits, upper and lower quartiles; whiskers, $1.5 \times$ interquartile range; points, outliers. d The topological representation of the expression space of LGG is labeled according to the prevalence of some of the significantly localized mutations. The three large expression groups in the topological representation are identified with oligodendrogliomas (enriched for CIC and IDH2 mutations), IDH1-mutant astrocytomas (enriched for TP53 mutations), and IDH1-wild-type astrocytomas (enriched for EGFR mutations). IDH1-mutant astrocytomas with a low G-C island methylation phenotype (G-CIMP low) form a flare of IDH1-wild-type astrocytomas. e Number of significant mutated genes as a function of the cohort size, for the original (red) and a randomized (blue) version of the LGG cohort. Our integrative topological approach produces significant results for tumor cohorts above 100 patients. $\mathbf{f}$ Comparison of the results of the integrative topological approach with those of MutSig2CV on the same cohort. Represented is the rank of each gene according to their significance in the topological (horizontal axis) and MutSig2CV (vertical axis) analyses in logarithmic scale. Genes that are significant ( $q$-value $<0.15$ ) according to our topological approach are marked in red. Genes below the red dashed line are significant ( $q$-value $<0.15$ ) according to the MutSig2CV analysis. Horizontal bars indicate the 16 and $84 \%$ percentiles of the ranks across the parameter space of the topological representation. Besides being radically different approaches, the results of our topological approach and MutSig2CV display a large degree of consistency, with most cancer-associated mutated genes sitting across the diagonal.

localized within the expression space of oligodendrogliomas, indicating a distinct expression profile from that of IDH1-mutant oligodendrogliomas (Fig. 1d, Supplementary Fig. 2a). This observation is consistent with a recent study based on genomic variations $^{31}$.

Neuronal marker expression has been reported in malignant (grade III/IV) gliomas other than classical anaplastic gangliogliomas 32,33 . In our cohort, tumors expressing canonical neuronal markers like neurofilament (NEFL, NEFM, and NEFH) and synaptophysin $(S Y P)$ were significantly localized within the expression space of oligodendrogliomas $(q$-value $<0.015$, Supplementary Fig. 3). These tumors harbored frequent deletions of the chromosome arm 19q, in addition to molecular alterations characteristic of astrocytic gliomas, such as TP53 and ATRX mutations (Fisher's exact test $p$-value $<0.01$, Supplementary Fig. 3). Although the average estimated tumor purity ${ }^{34}$ in this group was significantly lower than for the rest of the oligodendroglioma expression group (Mann-Whitney U-test $p$ value $=0.001$, average estimated tumor purities $=92$ and $96 \%$, respectively), the estimated tumor content was in many cases ( $n$ $=7$ ) above $98 \%$, suggesting that the expression of neuronal markers is not due to a poor tumor purity.

Computational benchmarking. To assess the number of cancerassociated genes identified by our approach as a function of the size of the cohort, we repeated the same analysis in smaller cohorts generated by randomly sampling patients from the original LGG cohort (Fig. 1e). We also assessed the number of false positives by generating randomized datasets, where we permuted the labels of the patients in the expression data. We observed that our approach requires a minimum cohort size of $\sim 100$ tumors. For larger cohorts, the expected number of false positives was between 1 and 2 (Fig. 1e).

Next, we sought to compare our results against current algorithms for the identification of cancer-associated genes using expression data. To that end, we analyzed the same LGG cohort using the recently published algorithm Xseq ${ }^{16}$ (Methods). Xseq implements a hierarchical Bayes statistical model to quantify the impact of somatic mutations on expression profiles using a precomputed 'influence graph' that encodes whether two genes are known to be functionally related. The analysis of the LGG cohort with Xseq led to only two significant genes (posterior probability, $P(D)>0.80)$, of which only one $(P T E N)$ has been previously reported in LGG. These results reveal the high sensitivity of our topological approach compared to state-ofthe-art algorithms.

In addition to Xseq, we compared the results of our integrative topological approach to those produced by MutSig2CV on the same cohort (Fig. 1f). MutSig2CV models the neutral background mutation rate, taking into account genomic variations due to differences in expression level and replication time ${ }^{3}$. We observed a significant overlap between the results of MutSig2CV and those of our approach, with 15 out of 23 mutated genes that were significant $(q$-value $<0.15)$ according to MutSig2CV, being also significant according to our approach (65\% overlap, Fisher's exact test $p$-value $=10^{-42}$ ). Some of the most significant cancer genes identified by MutSig2CV based on recurrence, such as PIK3R1 (mutated in $4 \%$ of the tumors), were not selected by our expression-based approach, highlighting the independence of recurrence- and expression-based approaches. Combining the results of MutSig2CV with those of our integrative topological approach (Fig. 1f) singled out new low-prevalence mutated genes, such as $\mathrm{NOTCH} 2$, as potential drivers of tumor progression in LGG.

Seeking a more systematic comparison with existing methods, we performed a similar study to that of Bertrand et al. ${ }^{35}$ across multiple tumor types (Methods). We estimated the precision, recall, and F1 score of our integrative topological approach, Xseq, MutSig2CV, OncodriveFML ${ }^{36}$, and $20 / 20+{ }^{37}$ based on the overlap of their top 15 predictions with a gold-standard list of cancer-associated genes ${ }^{35}$. In addition to the LGG cohort, we analyzed two cohorts of 208 colorectal adenocarcinoma (COAD) and 930 breast invasive carcinoma (BRCA) tumors from TCGA, respectively. In each of the three cohorts, the precision, recall, and F1 score of our integrative topological approach were the highest or second highest among the 5 algorithms (Supplementary Table 1), highlighting its utility for the identification of mutated cancer-associated genes.

Identification of cancer-associated genes across 12 cancers. Based on the above results, we decided to extend our analysis to other tumor types. We considered 12 tumor types from TCGA for which there were sufficient samples $(n>140)$ with RNA-seq and whole-exome data available (Table 1). The complete results 


\begin{tabular}{|c|c|c|c|}
\hline Cancer type & Cohort & Samples (TDA) & Samples (MutSig2CV) \\
\hline Bladder urothelial carcinoma & BLCA & 391 & 395 \\
\hline Breast invasive carcinoma & BRCA & 930 & 978 \\
\hline Cervical and endocervical cancers & CESC & 184 & 194 \\
\hline Colon adenocarcinoma & COAD & 208 & 367 \\
\hline Brain lower grade glioma & LGG & 513 & 516 \\
\hline Lung adenocarcinoma & LUAD & 470 & 533 \\
\hline Pheochromocytoma and paraganglioma & PCPG & 181 & 179 \\
\hline Stomach adenocarcinoma & STAD & 263 & 393 \\
\hline Testicular germ cell tumors & TGCT & 149 & 147 \\
\hline Thyroid carcinoma & THCA & 403 & 496 \\
\hline
\end{tabular}

of our analysis can be accessed through an online database (Methods). In total, our approach identified 95 mutated cancer genes $(q$-value $<0.15)$, out of which 16 genes were significant in two or more tumor types (Fig. 2a, Supplementary Figs. 4-15, and Supplementary Data 1). Some of the most common genes were TP53, KRAS, HRAS, PIK3CA, ATRX, EGFR, and NF1. The number of significant genes in each tumor type was correlated with the size of the cohort (Fig. 2b, Spearman's correlation coefficient $r=0.67, p$-value $=0.02$ ), consistently with the results of the computational benchmarking. We observed a large degree of consistency between the list of significant genes and curated databases of cancer genes. Specifically, $61 \%$ of the significant genes in our analysis were present in the Cancer Gene Census ${ }^{38}$ or OncoKB ${ }^{39}$ databases (Fig. $2 c$, Fisher's exact test $p$-value $<10^{-50}$ for each database). Overall, $75 \%$ of the patients carried a mutation in a significant gene, out of which $24 \%$ carried mutations in actionable genes with approved drugs ${ }^{35}$ (Supplementary Table 2).

The results were largely consistent with those of MutSig2 CV on the same TCGA cohorts (Fig. 2a, Supplementary Fig. 16, and Table 1), adding further support to some of the cancer genes identified in our analysis. Out of the 95 significant genes in the integrative topological analysis, 38 genes were not significant according to MutSig2CV (Fig. 2a, $q$-value $<0.15$ ). However, these putative elusive cancer genes did often displayed a tendency towards significance in the MutSig2CV analysis, likely reflecting a limitation of the cohort size (Supplementary Fig. 16). They also had a significant overlap with the Cancer Gene Census (eight out of 38 genes, Fisher's exact test $p$-value $=2 \times 10^{-5}$, Fig. $2 \mathrm{~d}$ ) and OncoKB (six out of 38 genes, Fisher's exact test $p$-value $=3 \times 10^{-4}$, Fig. $2 \mathrm{~d}$ ) databases, as well as with genes involved in developmental processes (27 out of 38 genes, g:SCS $q$-value $=10^{-3}$ ). Elusive genes included NOTCH2 mutations in breast invasive carcinoma (mutated in $2 \%$ of the tumors), which have been recently reported by manual inspection ${ }^{40}$; inactivating mutations of the tumor-suppressor genes KMT2A $A^{41}$ (also known as MLL1) and CUX1 ${ }^{42}$ in head and neck squamous cell carcinoma (each present in $1 \%$ of the tumors); inactivating mutations of the tumor-suppressor gene ADAMTS $12^{43}$ in lung adenocarcinoma (present in $4 \%$ of the tumors); mutations in the kinase domain of CHEK2 in thyroid carcinoma (present in $1 \%$ of the tumors), which have been associated with increased susceptibility to this cancer type ${ }^{44}$; inactivating mutations of the putative tumor-suppressor gene USP9X in thyroid carcinoma (present in $1 \%$ of the tumors), which codes for a deubiquitinase regulating the TGF $\beta^{45}$ and hippo signaling pathways ${ }^{46}$; and inactivating mutations of ATRX in pheochromocytoma and paraganglioma ${ }^{47}$ (present in $2 \%$ of the tumors). These genes, except CHEK2, encode long proteins
( $>1500$ amino acids) and are expected to contain numerous passenger mutations, complicating the identification of lowprevalence cancer-associated mutations using recurrence-based methods.

Additionally, the combination of the results of our analysis with those of MutSig2CV allowed us to prioritize the study of mutated genes in colon adenocarcinoma, where the number of significant genes according to MutSig2CV is too large $(n=1698$ genes, $q$-value $<0.15$ ) (Supplementary Fig. 16). In particular, our analysis highlighted ARHGAP5 and ARFGEF1 as previously unreported putative driver genes of tumor progression in this cancer type.

Truncating mutations of ADAMTS12 in lung adenocarcinoma. Using TCGA survival data we found that, among the previously unreported cancer-associated genes, inactivating mutations of ADAMTS12 were associated with poor survival (Fig. 3a). ADAMTS12 is a metalloproteinase with thrombospondin motif that can block the activation of the Ras-MAPK signaling pathway ${ }^{43}$. Immunodeficient mice injected with A549 lung adenocarcinoma (LUAD) cells overexpressing ADAMTS12 had a deficiency of tumor growth in comparison with tumors formed from parental A549 cells ${ }^{43}$. The ADAMTS12 gene is in chromosomal arm $5 p$, which is entirely amplified in over $60 \%$ of lung adenocarcinoma tumors ${ }^{48}$. It has been suggested that the TERT gene, coding for the telomerase catalytic subunit, may be the target of this amplification ${ }^{48}$. Consistent with the suggested antitumorgenic properties of ADAMTS12, we observed that LUAD patients with chromosome $5 p$ amplification and unaltered ADAMTS12 gene have better overall survival than those without chromosome $5 \mathrm{p}$ amplification (Fig. 3a, median overall survival 4.2 years versus 3.4 years, respectively, Kaplan-Meier $p$-value $=$ $0.05)$. To the contrary, patients with chromosome $5 p$ amplification and truncating mutations in ADAMTS12 have a reduced survival with respect to patients that harbor the amplification without mutations in ADAMTS12 (Fig. 3a, median overall survival 2.4 years, Kaplan-Meier $p$-value $=0.015)$. Additionally, truncating mutations in ADAMTS12 tend to co-occur with chromosome $5 \mathrm{p}$ amplification (Fig. 3a, one-tailed Fisher's exact test $p$-value $=2 \times 10^{-3}$ ).

To validate ADAMTS12 inactivation as a driver of progression in lung carcinoma, we investigated the effect of silencing ADAMTS12 in the lung carcinoma cell line LL/2-luc-M38 using a shRNA plasmid. In vitro proliferation and invasion assays revealed a significant increase in the proliferative and invasive potential of the cells that were transfected with the shRNA 

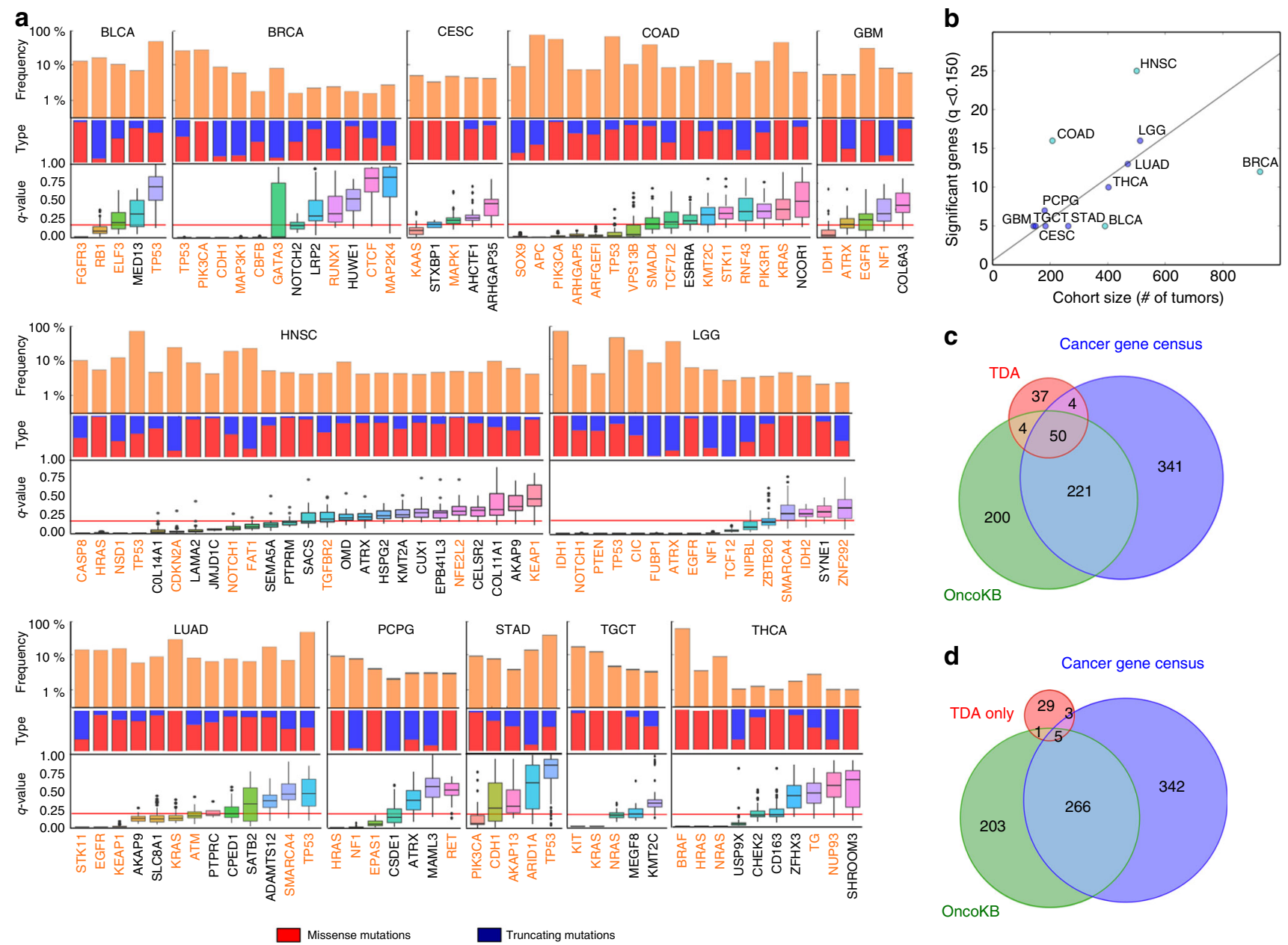

Fig. 2 Identification of mutated cancer genes across 12 tumor types using an integrative topological approach. a Significant genes (permutation test $q$ value $<0.15, n=10^{4}$ permutations, Benjamini-Hochberg procedure for multiple-hypothesis testing) in the integrative topological analysis of the 12 tumor types considered in Table 1. From top to bottom, the frequency of nonsynonymous mutations, the fraction of missense versus truncating mutations, and the distribution of $q$-values across the parameter space is shown for each gene. Genes that are also significant $(q$-value $<0.15)$ based on MutSig2CV are shown in orange. Box-plot elements: center line, median; box limits, upper and lower quartiles; whiskers, $1.5 \times$ interquartile range; points, outliers. b Plot of the number of significant genes ( $q$-value $<0.15$ ) against the number of tumors in the cohort, for each tumor type. A linear fit is shown, where outliers (marked in cyan) where not taken into account in the fit (Pearson's $r=0.94, p$-value $=5 \times 10^{-4}$ ). c Venn diagram showing the overlap between significant genes (TDA) and the curated databases of cancer genes OncoKB (two-tailed Fisher's exact test $p$-value $<10^{-50}$ ) and the Cancer Gene Census (two-tailed Fisher's exact test $p$-value $<10^{-50}$ ) over a background of 19,882 genes considered in the analysis. $\mathbf{d}$ Venn diagram showing the overlap between genes that are significant in the integrative topological analysis but not in the MutSig2CV analysis (TDA only), and the curated databases of cancer genes OncoKB (two-tailed Fisher's exact test $p$-value $=3 \times 10^{-4}$ ) and the Cancer Gene Census (two-tailed Fisher's exact test $p$-value $=2 \times 10^{-5}$ ) over a background of 19,882 genes considered in the analysis.

plasmid compared to the control cells (Fig. 3b, c, Mann-Whitney U-test $p$-value $<10^{-3}$ in both assays).

In addition to these in vitro studies, we assessed the effect of ADAMTS12 inactivation in vivo. To that end, we generated ADAMTS12-I- mice as previously described ${ }^{49}$ and treated ADAMTS12-knockout and control mice with urethane (ethyl carbamate), a carcinogen that typically induces lung adenomas after several months of treatment ${ }^{50,51}$. After 20 weeks of treatment, ADAMTS12-knockout mice showed a 5-fold enrichment on the number of lung tumors compared to control mice (Fig. 3d, Mann-Whitney U-test $p$-value $=3 \times 10^{-5}$ ). The enrichment in the number of tumors was still significant after disaggregating tumors by their size (Supplementary Fig. 17). We did not find a significant difference between the observed tumor size in control and ADAMTS12-knockout mice. Immunohistochemistry staining of tumor sections from these mice revealed some level of expression of ADAMTS12 in the region surrounding the adenoma, but not in the highly-proliferative Ki$67^{+}$cells (Supplementary Fig. 18). A similar pattern of ADAMTS12 expression has been observed in human colon adenocarcinoma ${ }^{52}$. Taken together our results suggest ADAMTS12 has a tumor-suppressor role in lung cancer, consistently with the results of our computational analysis.

\section{Discussion}

To identify which somatic mutations are relevant to the progression of tumors, most genomic analyses focus on the recurrence of mutations and define candidate cancer-associated genes as those mutated at a higher frequency than expected under a modeled local neutral mutation rate. This definition has proven to be particularly powerful for commonly mutated genes. However, it is limiting for low-prevalence mutations or tumors with a higher mutation burden. Here, we have adopted an alternative definition for candidate cancer-associated gene based on the 

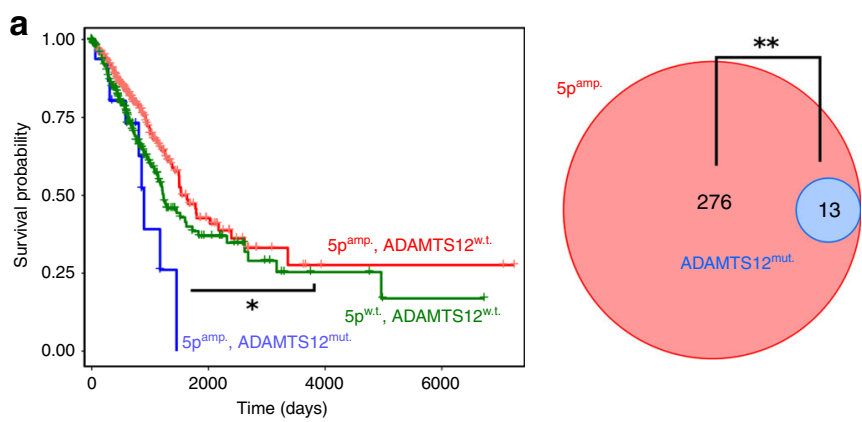

b

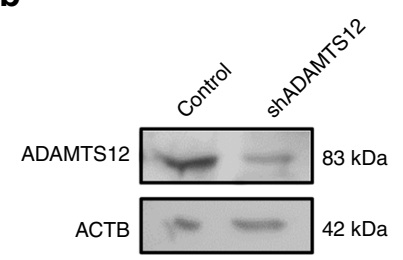

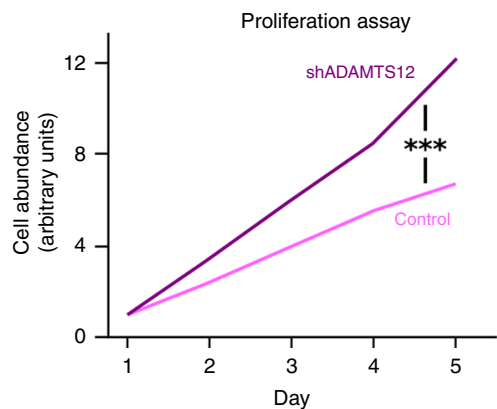

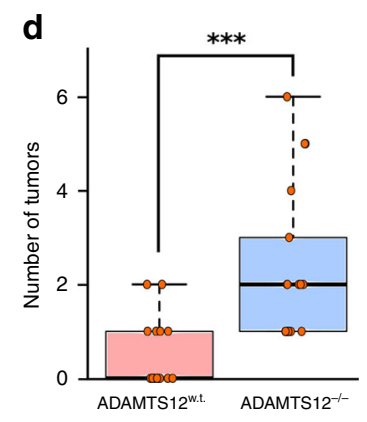

C

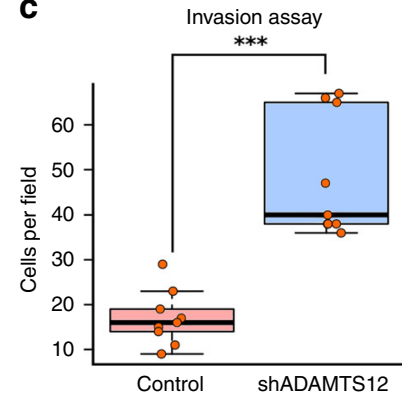

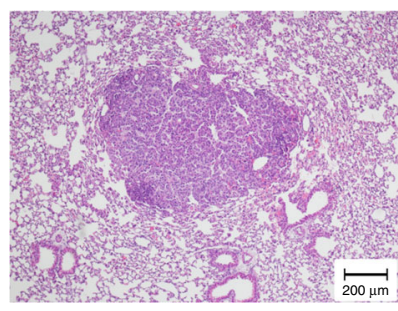

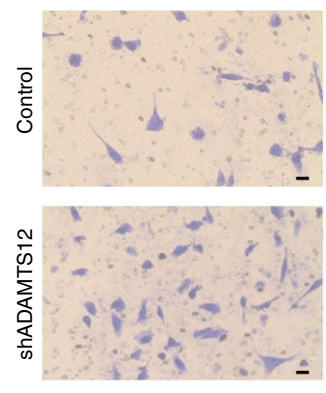

Fig. 3 Truncating mutations of ADAMTS12 are associated with increased tumor susceptibility and poor survival in LUAD. a Left: Kaplan-Meier survival curves for the LUAD cohort, where patients have been stratified according to whether their tumors have chromosome $5 p$ amplification and absence of truncating mutations in ADAMTS12 (red, $n=276$ patients), chromosome $5 p$ amplification and presence of truncating mutations in ADAMTS12 (blue, $n=13$ patients), and absence of both chromosome $5 p$ amplification and truncating mutations in ADAMTS12 (green, $n=181$ patients). Kaplan-Meier $p$-values of blue and green survival curves with respect to red survival curve are 0.015 and 0.05 , respectively. Right: Venn diagram showing the overlap between tumors with chromosome $5 p$ amplification (red) and tumors with truncating mutations in ADAMTS12 (blue) (one-tailed Fisher's exact test $p$-value $=2 \times 10^{-3}$ ). b Left: Western blot showing the expression of ADAMTS12 and $\beta$-Actin in control and shADAMTS12 LL/C-luc-M38 cells. Right: In vitro proliferation assay of control and shADAMTS12 cells. The asterisks $\left.{ }^{(\star \star}\right)$ denote two-tailed Mann-Whitney U-test $p$-value $<10^{-3}$. Experiments included in six replicates. $\mathbf{c}$ Left: Number of control and shADAMTS12 cells with invasive potential in an in vitro invasion assay $(n=4704$ cells in total examined over three biologically independent experiments with three replicates per condition). The asterisks ${ }^{\star * \star}$ ) denote two-tailed Mann-Whitney $\mathrm{U}^{- \text {test }} p$-value $<10^{-3}$. Right: Representative images of fields used for quantification after invasion assay. The size of the scale bar is $25 \mu \mathrm{m}$. $\mathbf{d}$ Left: Number of lung tumors observed in control $(n=17)$ and ADAMTS12-deficient $(n=17)$ mice treated with urethane after 20 weeks of treatment. ADAMTS12-deficient mice show a 5-fold enrichment in the number of lung tumors as compared to control mice. The asterisks ${ }^{* \star *}$ ) denote two-tailed Mann-Whitney $U$-test $p$-value $=3 \times 10^{-5}$. Right: Hematoxylin-eosin stained tissue section of ADAMTS12-deficient mice treated with urethane displaying a lung adenocarcinoma tumor. Box-plot elements: center line, median; box limits, upper and lower quartiles; whiskers, 1.5× interquartile range; points, outliers. Source data are provided as a Source Data file.

assumption that mutations in these genes are accompanied by consistent global expression patterns in the tumor. Remarkably, these two fundamentally different definitions are in practice highly consistent with each other, as we find that most mutations occurring at a high frequency compared to the local neutral mutation rate are associated with consistent global mRNA expression patterns in the tumor. As expected, there are numerous exceptions to this rule and utilizing our approach we are able to identify multiple candidate cancer genes that remained elusive to other methods. One example of such elusive cancerassociated mutations are truncating mutations of the PEST domain of $\mathrm{NOTCH} 2$ occurring in breast invasive carcinoma ${ }^{40}$. These rare events are easily masked by the large number of passenger mutations that this long gene accumulates. However, we find these alterations are consistently accompanied by global changes in the expression profile of the tumor. Although they affect a small fraction of all breast cancer patients, the availability of pharmacological inhibitors of the Notch signaling pathway makes them a promising therapeutic target for the treatment of these patients ${ }^{53}$. Among the less studied, elusive candidate cancer-associated mutations identified with our approach, we have studied the inactivating mutations of ADAMTS12 occurring in lung adenocarcinoma. We have provided evidence of the tumor-suppressor role of ADAMTS12 in this cancer type both in vitro and in vivo. Specifically, our experiments reveal that lung carcinoma LL/2-luc-M38 cells display a higher proliferative and invasive potential in vitro when transfected with an ADAMTS12 shRNA. Additionally, we have shown that mice treated with urethane have a several fold increase in the susceptibility to develop lung adenomas when ADAMTS12 is knocked out. These results are consistent with the observation that patients of lung adenocarcinoma with tumors harboring truncating mutations of ADAMTS12 have poor survival. Our work demonstrates that the combination of recurrence-based methods with integrative approaches as we describe here can be a valuable tool to systematically identify potentially actionable, lowprevalence mutations that escape standard methods of detection.

\section{Methods}

Sample collection and preprocessing. We collected gene expression levels and somatic mutation data of 12 tumor types from the TCGA repository (https://portal. gdc.cancer.gov/) (Table 1 and Supplementary Table 3). We only considered patients for which both types of data were available. RNA-seq expression levels were retrieved in RSEM format ${ }^{54}$ and estimated relative abundances $(x)$ were transformed according to the formula $r=\log _{2}\left(1+10^{6} \times x\right)$ for each gene. Curated somatic mutations were retrieved from the Broad Institute TCGA GDAC Firehose 
Portal (http://gdac.broadinstitute.org/). Gene names were adapted to comply with those in the NCBI Entrez ID database as of July 7, 2015.

Topological representations. We used the algorithm Mapper ${ }^{28}$, implemented in the Ayasdi software (https://www.ayasdi.com/platform/), to build topological representations of the RNA-seq data of each cancer cohort. Mapper builds upon any dimensional reduction algorithm (also known as "filter function") to produce a new low-dimensional network representation on which local relationships are preserved. To that end, Mapper covers the low-dimensional representation with overlapping bins and performs single-linkage clustering of the points in the highdimensional space. The number of bins and their overlap are specified by the "resolution" and "gain" parameters, respectively. The number of clusters in each bin is determined by the method described in Singh et al. ${ }^{28}$. A low-dimensional network is then built by assigning a node to each cluster, and if a sample appears in two nodes they are connected by an edge. A more detailed description of the Mapper algorithm for biologists can be found in the Methods section and Supplementary Note of Rizvi et al. ${ }^{25}$.

The output of Mapper is sensitive to several algorithmic choices. In our application, the following choices were made:

Metric: We used Pearson's correlation distance using the top 4500 genes with highest variance as a measure of the similarity among the expression profile of tumors. We did not observed substantial differences between using Pearson's and Spearman's correlation distance in our analyses. We therefore used Pearson's correlation distance given its reduced computation time in large datasets.

Filter function: We built a $k=30$ nearest neighbors graph using Pearson's correlation distances between the samples and used a 2-dimensional embedding of the shortest path distances on this graph as the filter function. This choice filter function was based on the ability to capture biological proxies, such as the separation between the expression profiles of normal and tumor samples and the identification of known driver genes. Other choices of 2-dimensional filter functions, such as Principal Component Analysis (PCA), Multidimensional Scaling, or Uniform Manifold Approximation and Projection (UMAP) ${ }^{55}$ led to consistent results. For example, Pearson's correlation coefficient between the $C$ (g) scores (defined below) computed in Mapper representations of the LGG expression space using 2-dimensional embedding of the shortest path distance or PCA as filter functions was $r=0.88$ (test for association $p$-value $=2 \times 10^{-4}$ ), whereas using UMAP as filter function was $r=0.93$ (test for association $p$ value $=10^{-5}$ ).

Resolution and gain parameters: We covered the low-dimensional representation with overlapping squared bins. We scanned across the entire resolution and gain parameter space of the cover, as described in the paragraph "Parameter scan and selection", obtaining stable results. For example, the median Pearson's correlation coefficient between the $C(g)$ scores computed by varying the resolution parameter between 55 and 85 and the gain parameter between 6 and 9 in the Mapper representation of the LGG cohort expression space was $r=0.97$ (test for association $p$-value $<10^{-15}$ ).

Statistical analysis. We used the notions of topological association introduced in Rizvi et al. ${ }^{25}$ to identify features associated with localized regions of a phenotypic space. Our approach is similar to the Laplacian score of He, Cai, and Niyogi ${ }^{66,57}$, and complementary to other statistical methods for network analysis ${ }^{58}$. In our case, the features that we tested were the somatic mutations in the tumor cohort, and the phenotypic space was the expression space of the tumor cohort. For each mutated gene $g$ in the cohort, we considered the following score:

$$
C(g)=\frac{N}{N-1} \frac{\sum_{i, j \in \Gamma} e_{i}(g) A_{i j} e_{j}(g)}{\left(\sum_{k \in \Gamma} e_{k}(g)\right)^{2}}
$$

where $\Gamma$ denotes the set of nodes in the topological representation, $A_{i j}$ its adjacency matrix, $N$ the number of nodes in the representation, and $e_{i}(g)$ the average frequency of nonsynonymous mutations of $g$ for the samples in node $i$. The score $C(g)$ is therefore a sum over the edges of the network, where the contribution of each edge is proportional to the product of the fraction of tumors that harbor the mutated gene in each of the two nodes connected by the edge. Qualitatively, this nonparametric score assesses the degree of similarity in gene expression for samples that carry a specific mutated gene. In our setting, it offers some advantages compared to straight nonparametric approaches like computing the variance in expression space for the samples that carry the mutated gene. In particular, since it is based on the graph distance, it can be safely used with nonlinear spaces, such as the gene expression spaces spanned by tumor cohorts. Its application to dimensionally reduced network representations that reduce the sparsity of the data, such as Mapper representations, is straightforward. Moreover, being a local approach, it is relatively insensitive to outliers.

To be able to compare the score of mutated genes with different prevalence, we introduced a permutation test for each gene. A null distribution was built for $C(g)$ by randomly permuting the patient id's in the exome data and a $p$-value was assigned to the score of each gene $g$ according to its null distribution. We performed $10^{4}$ permutations to build the null distribution of each gene. We controlled the false discovery rate (FDR) using the Benjamini-Hochberg (BH) procedure ${ }^{59}$. To avoid too large corrections due to multiple-hypothesis testing, we only considered mutated genes with a prevalence in the cohort above a given threshold. The thresholds used in each cohort are summarized in Supplementary Table 3. In addition, we limited the number of genes in each analysis to the 350 genes with highest ratio between nonsynonymous and total number of mutations. These thresholds were empirically determined for each cohort by looking at the size of the BH correction that resulted at different choices of the thresholds. For some cancer types, avoiding a large BH correction required relatively stringent thresholds (Supplementary Table 3), possibly reflecting noisier expression networks, e.g., due to differences in tumor purity among patients.

Parameter scan and selection. To optimize the sensitivity of our approach at a fixed false discovery rate and control for the stability of the results against parameter choices, we generated 49 topological representations for the expression data of each tumor type by scanning over the parameter space of the Mapper algorithm. The resolution parameter was taken in the range 10-80, in intervals of 10 , and the gain parameter 1.5-8.5, in intervals of 1. For each topological network, the statistical analysis performed in the previous paragraph was performed independently. We then selected a finer region in the parameter space for each cohort based on the following criteria:

A large number of mutated genes with a significant score $(q$-value $<0.15)$ at a fixed FDR.

Absence of significant spurious correlations and batch effects (as described in next paragraph).

For each selected region in the parameter space, we performed a finer scan across the resolution and gain parameters, taking intervals of 5 and 0.5 , respectively.

Control of spurious associations with expression. Hypermutated tumors often have a distinctive expression signature. In those cases, some localized regions of the expression space will consist of tumors with a higher mutation rate. Those localized regions will harbor an accumulation of passenger mutations that may confound our approach. To control for associations between global expression patterns and the tumor mutation rate, we assessed the localization of the mutational tumor burden on the topological representations using the same approach as described in the paragraph "Statistical analysis", where $e_{i}$ is now the average frequency of somatic mutations for the samples in node $i$. If the localization of the mutational burden was significant ( $p$-value $<0.05$ ), we manually set a threshold on the mutational burden to split the cohort into hypermutated and nonhypermutated tumors. This process could have been automated; however, we found it unnecessary as small changes in the threshold do not affect substantially the results. The thresholds used in each cohort are summarized in Supplementary Table 3. We randomly subsampled mutations from each of the hypermutated tumors so that after subsampling the median mutational burden for hypermutated tumors in the cohort was equal to the median mutational burden for nonhypermutated tumors. We reassessed the significance of the localization of the mutational burden using the down sampled data. If the degree of localization of the mutational tumor burden was not significant, we continued the analysis using the down sampled mutation data. Otherwise, if the degree of localization was still significant after subsampling, we did not include the cohort in our study.

To control for associations between expression and mutation rates within the same gene, such as those due to transcription-coupled DNA repair, we assessed the similarity between the profiles of somatic mutation and mRNA expression on the topological representations. To that end, we computed the Jensen-Shannon divergence between the expression and mutation profiles of each gene in the topological representations using the formula

$$
J(g)=\frac{1}{2} \sum_{i \in \Gamma}\left[-\left(\tilde{e}_{i}(g)+\tilde{r}_{i}(g)\right) \log \left(\frac{\tilde{e}_{i}(g)+\tilde{r}_{i}(g)}{2}\right)+\tilde{e}_{i}(g) \log \left(\tilde{e}_{i}(g)\right)+\tilde{r}_{i}(g) \log \left(\tilde{r}_{i}(g)\right)\right]
$$

where $\tilde{e}_{i}(g)$ and $\tilde{r}_{i}(g)$ are, respectively, the fraction of tumors with gene $g$ somatically mutated and the average expression of gene $g$ in the tumors associated with the $i$-th node of the topological representation, normalized such that

$$
\sum_{i \in \Gamma} \tilde{e}_{i}(g)=\sum_{i \in \Gamma} \tilde{r}_{i}(g)=1
$$

The significance of $J(g)$ was assessed for each gene independently by means of a permutation test. To that end, for each gene the labels of the samples on the mutation data were randomly permuted 2000 times, and $J(g)$ was computed in each permutation. A $p$-value was estimated by counting the fraction of permutations that led to a value of $J(g)$ smaller than the original value. Genes with a $p$-value for $J$ $(g)$ closed to 0 displayed a large degree of correlation between expression and mutation in the topological representation, whereas genes with a $p$-value for $J(g)$ closed to 1 displayed a large degree of anti-correlation between expression and mutation in the topological representation. After adjusting for multiple-hypothesis testing using Benjamini-Hochberg procedure to control the false discovery rate, we removed genes from the analysis for which the median $q$-value for $J(g)$ across the parameter space of the topological representation was above 0.8 , as those are 
potentially related to spurious anticorrelations between gene expression and mutation.

Last, to control for the presence of batch effects due to differences among mutation calling centers, we assessed the degree of localization of batches in the topological representation using the same approach as described in the paragraph "Statistical analysis", with $e_{i}$ now represents the fraction of tumors in node $i$ that were processed by a given center. We removed the contribution of batches whose degree of localization was significant $(p$-value $<0.05)$ according to this procedure.

Computational benchmarking. We generated smaller LGG datasets by randomly sampling 50,100, 200, 300, and 400 patients from the original LGG cohort. For each of these sizes, we generated a null dataset by randomly permuting the labels of the patients on the expression data. We ran the integrative topological analysis in each of these new datasets using the same parameters than in the original analysis of the LGG cohort (Supplementary Table 3).

To benchmark the performance of algorithms based on a gold-standard list of cancer-associated genes, we followed the same approach as in Bertrand et al. ${ }^{35}$. We considered the same gold-standard list as in that reference. For each of the algorithms evaluated, we computed the precision (P), recall (R), and F1 score based the top $\min (15, G)$ significant genes $(q$-value $<0.15)$

$$
P=\frac{T}{\min (15, G)}, \quad R=\frac{T}{15}, \quad F 1=2 \cdot \frac{P \cdot R}{P+R}
$$

where $G$ is the total number of significant genes and $T$ the number of top $\min (15$, $G)$ significant genes present in the gold-standard. We run Xseq, OncodriveFML and $20 / 20+$ with default parameters, as described in their documentation.

MutSig2CV analyses. We downloaded from the Broad Institute TCGA GDAC Firehose Portal (http://gdac.broadinstitute.org/) the MutSig2CV v3.1 analyses of each of the 12 TCGA cohorts (Supplementary Table 3).

Online database. Representative topological representations and precomputed statistics were deposited in an online database for each of the 12 tumor types considered in this study (https://rabadan.c2b2.columbia.edu/pancancer). The interface of the database allows to explore the results of the analysis interactively.

Induction of lung tumors in mice. Mouse experiments were performed following the institutional guidelines of the University of Oviedo (Comité de Ética en Experimentación Animal). Adamts $12^{-/-}$mice were generated in a C57BL/6 genetic background and genotyped as in El Hour et al. ${ }^{49}$. Lung tumors were induced in 6-8-weeks-old mice by intraperitoneal injection of eight doses of $1 \mathrm{~g} / \mathrm{kg}$ of urethane (ethyl carbamate; Sigma); second dose was given $48 \mathrm{~h}$ after the initial one and then once a week to reach a total of eight doses. Mice were fed ad libitum in standard housing conditions and sacrificed 20 weeks after the first urethane injection. Left lungs were fixed in $4 \%$ paraformaldehyde, paraffin-embedded and sectioned every $100 \mu \mathrm{m}$ in of $10-\mu \mathrm{m}$ slices. These were then stained with hematoxylin/eosin for morphological examination by experienced pathologists (Unidad de Histopatología Molecular en Modelos Animales de Cáncer, IUOPA). Tumors were quantified and classified according to their diameter in large $(>400 \mu \mathrm{m})$, medium $(200-400 \mu \mathrm{m})$, and small $(<200 \mu \mathrm{m})$ tumors.

Generation of shADAMTS12 LL/2-luc-M38 cells. We used an Adamts12 Mouse shRNA Plasmid (OriGene, Locus ID: 239337) and transfected LLC/2-luc-M38 (Caliper) cells with lipofectamine/plus (ThermoFisher Scientific) following the recommendations of manufacturer. We checked transfected cells for ADAMTS12 expression by western blot for ADAMTS12 (Santa Cruz Biotechnology H-142; $1: 200$ dilution) and $\beta$-actin (Sigma-Aldrich AC-15; $1: 5000$ dilution) in $10 \%$ polyacrylamide gels. Immunoreactive proteins were visualized using HRP-peroxidaselabeled anti-rabbit or anti-mouse secondary antibodies (Cell Signaling Technology 7074 S and Jackson ImmunoResearch 115-035-062; 1:10000 dilutions) and the ECL detection system (Pierce).

Proliferation assay. Cell proliferation was measured using the CellTiter 96 Nonradiactive Cell Proliferation Assay kit (Promega). LL/2-luc-M38 cells $\left(3 \times 10^{4} /\right.$ well) were seeded into 96 -well plates in six replicates. Cell proliferation rates were determined on five consecutive days using the automated microtiter plate reader Power Wave WS (BioTek).

Invasion assay. In vitro invasion potential was assessed using 24-well Matrigelcoated invasion chambers with $8 \mu \mathrm{m}$ pore size (BD Biosciences). A total of $5 \times 10^{4}$ cells were allowed to migrate for $24 \mathrm{~h}$ using $10 \%$ fetal bovine serum as chemoattractant. Cells that reached the lower surface were stained with crystal violet. At least three independent experiments were performed with triplicates for each condition. Cells were counted in eight randomly selected microscopic fields.

Immunohistochemistry. Lungs were fixed in $4 \%$ formalin for $24 \mathrm{~h}$. After fixation, samples were dehydrated and embedded in paraffin. Sections $4-\mu \mathrm{m}$ thick were stained with hematoxylin and eosin for microscopy examination and consecutive sections were used for immunohistochemical labeling. Sections were incubated with anti-ADAMTS12 (H-142, Santa Cruz Biotechnologies, $1 \mathrm{~h}$ at $37^{\circ} \mathrm{C}$; $1: 50$ dilution) or with anti-Ki-67 (ab66155, Abcam, o/n at $4{ }^{\circ} \mathrm{C} ; 1: 4000$ dilution) primary antibodies. Sections were then incubated 30 min with EnVision ${ }^{\text {TM }}+/$ HRP (Dako) and 5 min with Liquid DAB (Dako). Samples were counterstained with hematoxilin.

Reporting summary. Further information on research design is available in the Nature Research Reporting Summary linked to this article.

\section{Data availability}

The gene expression data that support the results of this study are available at the TCGA repository (https://portal.gdc.cancer.gov/). The complete list of RSEM files used from this portal can be found in Supplementary Table 3. The somatic mutation data and MutSig2CV results that support the results of this study are available at the Broad Institute TCGA GDAC Firehose Portal (http://gdac.broadinstitute.org/). The complete list of MAF and MutSig2CV files used from this portal can be found in Supplementary Table 3. Representative topological representations have been deposited in an online database for each of the 12 tumor types (https://rabadan.c2b2.columbia.edu/ pancancer). Source data are provided with this paper.

\section{Code availability}

The source code and scripts used in the paper have been deposited in GitHub (https:// github.com/CamaraLab/TDA-TCGA/).

Received: 30 August 2018; Accepted: 9 July 2020;

Published online: 30 July 2020

\section{References}

1. Garraway, L. A. \& Lander, E. S. Lessons from the cancer genome. Cell 153, 17-37 (2013).

2. Vogelstein, B. et al. Cancer genome landscapes. Science 339, 1546-1558 (2013).

3. Lawrence, M. S. et al. Mutational heterogeneity in cancer and the search for new cancer-associated genes. Nature 499, 214-218 (2013).

4. Lawrence, M. S. et al. Discovery and saturation analysis of cancer genes across 21 tumour types. Nature 505, 495-501 (2014).

5. Imielinski, M. et al. Mapping the hallmarks of lung adenocarcinoma with massively parallel sequencing. Cell 150, 1107-1120 (2012).

6. Rubino, G. \& Tuffin, B. Rare event simulation using Monte Carlo methods (John Wiley \& Sons, 2009).

7. De Haan, L. \& Ferreira, A. Extreme value theory: an introduction (Springer Science \& Business Media, 2007).

8. Cheng, F., Zhao, J. \& Zhao, Z. Advances in computational approaches for prioritizing driver mutations and significantly mutated genes in cancer genomes. Brief. Bioinform. 17, 642-656 (2016).

9. Thingholm, L. B. et al. Strategies for integrated analysis of genetic, epigenetic and gene expression variation in cancer: addressing the challenges. Front. Genet. 7, 2 (2016).

10. Ding, L. et al. Somatic mutations affect key pathways in lung adenocarcinoma Nature 455, 1069-1075 (2008).

11. Leary, R. J. et al. Integrated analysis of homozygous deletions, focal amplifications, and sequence alterations in breast and colorectal cancers. Proc. Natl Acad. Sci. USA 105, 16224-16229 (2008).

12. Frattini, V. et al. The integrated landscape of driver genomic alterations in glioblastoma. Nat. Genet. 45, 1141-1149 (2013).

13. Bashashati, A. et al. DriverNet: uncovering the impact of somatic driver mutations on transcriptional networks in cancer. Genome Biol. 13, R124 (2012).

14. Bertrand, D. et al. Patient-specific driver gene prediction and risk assessment through integrated network analysis of cancer omics profiles. Nucleic Acids Res. 43, e44 (2015).

15. Manolakos, A., Ochoa, I., Venkat, K., Goldsmith, A. J. \& Gevaert, O. CaMoDi a new method for cancer module discovery. BMC Genomics 15, S8 (2014)

16. Ding, J. et al. Systematic analysis of somatic mutations impacting gene expression in 12 tumour types. Nat. Commun. 6, 8554 (2015).

17. Giacomini, C. P. et al. A gene expression signature of genetic instability in colon cancer. Cancer Res. 65, 9200-9205 (2005).

18. Banerjea, A. et al. Colorectal cancers with microsatellite instability display mRNA expression signatures characteristic of increased immunogenicity. Mol. Cancer 3, 21 (2004).

19. Carlsson, G. Topology and data. Bull. Am. Math. Soc. 46, 255-308 (2009) 
20. Cámara, P.G. Topological methods for genomics: present and future directions. Curr. Opin. Syst. Biol. 1, 95-101 (2017).

21. Ceccarelli, M. et al. Molecular profiling reveals biologically discrete subsets and pathways of progression in diffuse glioma. Cell 164, 550-563 (2016).

22. Chan, J. M., Carlsson, G. \& Rabadan, R. Topology of viral evolution. Proc. Natl Acad. Sci. USA 110, 18566-18571 (2013).

23. Camara, P. G., Rosenbloom, D. I., Emmett, K. J., Levine, A. J. \& Rabadan, R. Topological data analysis generates high-resolution, genome-wide maps of human recombination. Cell Syst. 3, 83-94 (2016).

24. Camara, P., Levine, A. \& Rabadan, R. Inference of ancestral recombination graphs through topological data analysis. PLoS Comput. Biol. 12, e1005071 (2016).

25. Rizvi, A.H. et al. Single-cell topological RNA-seq analysis reveals insights into cellular differentiation and development. Nat. Biotechnol. 35, 551-560 (2017).

26. Nicolau, M., Levine, A. J. \& Carlsson, G. Topology based data analysis identifies a subgroup of breast cancers with a unique mutational profile and excellent survival. Proc. Natl Acad. Sci. USA 108, 7265-7270 (2011).

27. $\mathrm{Li}, \mathrm{L}$. et al. Identification of type 2 diabetes subgroups through topological analysis of patient similarity. Sci. Transl. Med. 7, 311 ra174 (2015).

28. Singh, G., Mémoli, F. \& Carlsson, G.E. in SPBG 91-100 (Citeseer, 2007).

29. Friedman, J., Hastie, T. \& Tibshirani, R. The Elements of Statistical Learning (Springer series in statistics New York, 2001).

30. Cancer Genome Atlas Research, N. et al. Comprehensive, integrative genomic analysis of diffuse lower-grade gliomas. N. Engl. J. Med. 372, 2481-2498 (2015).

31. Bolouri, H., Zhao, L. P. \& Holland, E. C. Big data visualization identifies the multidimensional molecular landscape of human gliomas. Proc. Natl Acad. Sci. USA 113, 5394-5399 (2016).

32. Kim, H. R. et al. Malignant glioma with neuronal marker expression: a clinicopathological study of 18 cases. J. Korean Neurosurg. Soc. 59, 44-51 (2016).

33. Varlet, P. et al. New variants of malignant glioneuronal tumors: a clinicopathological study of 40 cases. Neurosurgery 55, 1377-1391 (2004).

34. Aran, D., Sirota, M. \& Butte, A. J. Systematic pan-cancer analysis of tumour purity. Nat. Commun. 6, 8971 (2015).

35. Bertrand, D. et al. ConsensusDriver improves upon individual algorithms for predicting driver alterations in different cancer types and individual patients. Cancer Res. 78, 290-301 (2018).

36. Mularoni, L., Sabarinathan, R., Deu-Pons, J., Gonzalez-Perez, A. \& LopezBigas, N. OncodriveFML: a general framework to identify coding and noncoding regions with cancer driver mutations. Genome Biol. 17, 128 (2016).

37. Tokheim, C. J., Papadopoulos, N., Kinzler, K. W., Vogelstein, B. \& Karchin, R. Evaluating the evaluation of cancer driver genes. Proc. Natl Acad. Sci. USA 113, 14330-14335 (2016).

38. Futreal, P. A. et al. A census of human cancer genes. Nat. Rev. Cancer 4, 177-183 (2004).

39. Chakravarty, D. et al. OncoKB: a precision oncology knowledge base. JCO Precis. Oncol. 1, 1-16 (2017).

40. Wang, K. et al. PEST domain mutations in Notch receptors comprise an oncogenic driver segment in triple-negative breast cancer sensitive to a gamma-secretase inhibitor. Clin. Cancer Res. 21, 1487-1496 (2015).

41. Rao, R. C. \& Dou, Y. Hijacked in cancer: the KMT2 (MLL) family of methyltransferases. Nat. Rev. Cancer 15, 334-346 (2015).

42. Wong, C. C. et al. Inactivating CUX1 mutations promote tumorigenesis. Nat. Genet 46, 33-38 (2014).

43. Llamazares, M. et al. The ADAMTS12 metalloproteinase exhibits antitumorigenic properties through modulation of the Ras-dependent ERK signalling pathway. J. Cell Sci. 120, 3544-3552 (2007).

44. Cybulski, C. et al. CHEK2 is a multiorgan cancer susceptibility gene. Am. J. Hum. Genet. 75, 1131-1135 (2004).

45. Dupont, S. et al. FAM/USP9x, a deubiquitinating enzyme essential for TGFbeta signaling, controls Smad4 monoubiquitination. Cell 136, 123-135 (2009).

46. Thanh Nguyen, H. et al. Deubiquitylating enzyme USP9x regulates hippo pathway activity by controlling angiomotin protein turnover. Cell Discov. 2, 16001 (2016)

47. Fishbein, L. et al. Whole-exome sequencing identifies somatic ATRX mutations in pheochromocytomas and paragangliomas. Nat. Commun. 6, 6140 (2015).

48. Weir, B. A. et al. Characterizing the cancer genome in lung adenocarcinoma. Nature 450, 893-898 (2007).

49. El Hour, M. et al. Higher sensitivity of Adamts12-deficient mice to tumor growth and angiogenesis. Oncogene 29, 3025-3032 (2010).

50. Folgueras, A. R., Freitas-Rodriguez, S., Espanol, Y. \& Velasco, G. Cancer susceptibility models in protease-deficient mice. Methods Mol. Biol. 1731 235-245 (2018).
51. Miller, Y. E. et al. Induction of a high incidence of lung tumors in C57BL/6 mice with multiple ethyl carbamate injections. Cancer Lett. 198, 139-144 (2003).

52. Moncada-Pazos, A. et al. The ADAMTS12 metalloprotease gene is epigenetically silenced in tumor cells and transcriptionally activated in the stroma during progression of colon cancer. J. Cell Sci. 122, 2906-2913 (2009).

53. Yuan, X. et al. Notch signaling: an emerging therapeutic target for cancer treatment. Cancer Lett. 369, 20-27 (2015).

54. Li, B. \& Dewey, C. N. RSEM: accurate transcript quantification from RNA-Seq data with or without a reference genome. BMC Bioinforma. 12, 323 (2011).

55. Becht, E. et al. Dimensionality reduction for visualizing single-cell data using UMAP. Nat. Biotechnol. 37, 38-44 (2019).

56. He, X., Cai, D. \& Niyogi, P. Laplacian score for feature selection. in Advances in neural information processing systems 507-514 (2006).

57. Govek, K. W., Yamajala, V. S. \& Camara, P. G. Clustering-independent analysis of genomic data using spectral simplicial theory. PLoS Comput. Biol. 15, e1007509 (2019).

58. Kolaczyk, E.D. Statistical Analysis of Network Data: Methods and Models, (Springer Science \& Business Media, 2009).

59. Benjamini, Y. \& Hochberg, Y. Controlling the false discovery rate: a practical and powerful approach to multiple testing. J. R. Stat. Soc. Ser. B 57, 289-300 (1995).

\section{Acknowledgements}

We thank J. A. Vega and IUOPA (Servicio de Histopatología Molecular en Modelos Animales de Cáncer) for assistance with the histopathology of mouse tumors; R. Aubin, G. Carlsson, K. Govek, A. Iavarone, A. Lasorella, S. Lowe, F. Sanchez-Rivera, E. Troisi and S. Woodhouse for scientific discussions; and S. Christen, D. Ramanan, I. Sagalovskiy, and S. Thuault-Restituito for technical and administrative support. R.R. acknowledges funding from NIH (U54 CA193313-01). P.G.C. acknowledges funding from Stand-UpTo-Cancer Convergence 2.0.

\section{Author contributions}

P.G.C. and R.R. conceived the methodology. U.R. and A.N.A. implemented the computational pipeline. U.R. built the topological representations. Y.M., S.C., and A.J.O. conceived and performed the experiments. T.C. and O.E. designed and implemented the online database. A.J.L. and L.A. contributed to the analysis. All authors discussed the results and implications and collaborated on the writing of the manuscript.

\section{Competing interests}

The authors declare no competing interests.

\section{Additional information}

Supplementary information is available for this paper at https://doi.org/10.1038/s41467 020-17659-7.

Correspondence and requests for materials should be addressed to R.R., A.J.L. or P.G.C.

Peer review information Nature Communications thanks Denis Bertrand, Christopher Kemp and the other, anonymous, reviewer(s) for their contribution to the peer review of this work.

Reprints and permission information is available at http://www.nature.com/reprints

Publisher's note Springer Nature remains neutral with regard to jurisdictional claims in published maps and institutional affiliations.

Open Access This article is licensed under a Creative Commons Attribution 4.0 International License, which permits use, sharing, adaptation, distribution and reproduction in any medium or format, as long as you give appropriate credit to the original author(s) and the source, provide a link to the Creative Commons license, and indicate if changes were made. The images or other third party material in this article are included in the article's Creative Commons license, unless indicated otherwise in a credit line to the material. If material is not included in the article's Creative Commons license and your intended use is not permitted by statutory regulation or exceeds the permitted use, you will need to obtain permission directly from the copyright holder. To view a copy of this license, visit http://creativecommons.org/ licenses/by/4.0/

(C) The Author(s) 2020 